\title{
Morphological characterization and identification of two sesame (Sesamum spp.) ecotypes in Malaysia
}

\begin{abstract}
Malaysia is endowed with favourable ecologies for sesame (Sesamum spp. L.) cultivation but lack of information on genetic variations among Malaysian sesame populations has been a barrier to select the desirable accessions for commercial cultivation. Present study was done to characterize and identify the level of variations among 30 accessions of two sesame ecotypes collected from Port Dickson and Serdang, by using 39 morphological (19 quantitative and 20 qualitative) traits. Descriptive analysis showed a great amount of similarity for 31 morphological traits, while 8 quantitative traits found to be significantly varied - as for stem circumference, branching, flower number and size, pedicel length, ovary size, capsule number and size. In clustering analysis, the populations of both locations tended to cluster in a main group according to their qualitative traits similarity, but separated in two groups based on quantitative traits, indicating that quantitative traits are the dominant trait for separations of accessions. However, the dendogram developed in this study revealed four distinct clusters in which most of the accessions between two ecotypes were closely related with a similarity co-efficient ranged from 0.744 to 0.487 . Finally, all the accessions were to be considered as Sesamum radiatum due to the matching of capsule and seed characteristics with the taxonomic key developed for the identification of specific sesame species. The study therefore suggests that morphological variability observed in both populations is mainly attributed to differential habitat and soil nutrient conditions rather than genotypic diversity.
\end{abstract}

Keyword: Genetic variation; Sesame accessions; Sesamum spp; Morphological traits; Malaysia 